\title{
Breast Augmentation Virtual Surgery Using 3D Body Scanning Bridging the Gap between Patient Expectations and Surgical Practicalities
}

\author{
David A. GILBERT ${ }^{\mathrm{a}}$, David B. STEFAN ${ }^{\mathrm{b}}$ \\ ${ }^{a}$ The Hague Plastic and Cosmetic Surgery Center, Norfolk, Virginia, USA; \\ ${ }^{\mathrm{b}}$ Novaptus Systems Inc., Chesapeake, Virginia, USA \\ http://dx.doi.org/10.15221/13.285
}

Breast augmentation is an example of cosmetic surgery. The patient has a preconceived idea of what her ideal bust size would like to be. Typically, the images in her mind are derived from magazines, television shows and perhaps friends who have had the surgical procedure. She generally thinks of bust cup sizes. The surgeon, on the other hand, thinks of bust volumes in terms of cc's and implant sizes. This dislocation between the surgeon and the patient often leads to dissatisfaction of surgical results, mainly due to a lack of a common language. A technique in use today bridges patient expectations with surgical outcomes.

\section{Background}

The cosmetic surgeon faces uncertainty in predicting successful breast augmentation outcomes. From a purely surgical point of view, understanding pre-operative breast contours, assessing preoperative breast asymmetry and determining skin elasticity and muscle tone are all parts of the preoperative breast augmentation evaluation. The surgeon, through training and experience considers the subject's medical suitability for certain types of breast implants and has an idea of what implant sizes to suggest. The surgeon might even indicate that a procedure such as a mastoplexy be combined with surgical implants to achieve a desired result.

If the cosmetic surgeon happened to be consulting with another cosmetic surgeon, the communication between the two regarding the above paragraph would be direct, with little room for equivocation or misunderstanding. This is because the language between the two surgeons is common. They are trained in the medical sciences and medical arts, understand anatomical terms and surgical procedures

When two surgeons are asked to evaluate the same preoperative patient, though the language between the two is common, it is not uncommon to have two surgeons express different opinions as to which surgical procedure to use and which implant size to select that will achieve the best surgical outcome. This isn't necessarily because one surgeon is more comfortable and has more experience using a particular surgical technique.

This is because we have entered the realm of aesthetics.

The Oxford American dictionary defines aesthetics as: a). The branch of philosophy that deals with the nature and expression of beauty, b). In Kantian philosophy, the branch of metaphysics concerned with the laws of perception.

What the surgeon, or both surgeons are, in effect doing is placing a value judgment on the expected aesthetic outcome of the surgical procedure, from their point of view. And both surgeons are viewing the potential outcome from their surgical procedure comfort level, their knowledge of implant size cc's, types of implants and whether to use potential overfill of an implant to compensate for any perceived asymmetries, or other surgical techniques that may need to be implemented during the course of surgery.

The patient, on the other hand, is not really concerned about the particular surgical implementation, other than the potential scarring effects. She's not necessarily concerned about the implant size, or even the implant type though the choice of which are integral to the surgical outcome. She has an image in her mind of her ideal bust size after surgery. The outcome, to her, can be expressed in bust and bra cup size and whether the surgery overcame any preoperative issues such as ptotic breasts, asymmetry or other condition she considered to be deficient in some way.

From this perspective, it is easy to see why patient dissatisfaction can arise from what the surgeon would consider to be an excellent outcome. Dissatisfaction, in this case, does not necessarily mean wholly dissatisfied. Rather, it can manifest itself in disappointment, with thoughts like "What would the results have been if I had chosen a larger implant"? Or, "What if I went with a gel, high profile implant rather than the lower profile saline implant"? 
The root cause of such potential disappointment often comes down to the lack of common language, or understanding between what the patient perceives she desires, and what the surgeon, given the patient's desires, can achieve.

\section{The Traditional Breast Augmentation Consultation}

Perhaps the most difficult part of the process is gauging patient expectations. What does she want? Does she want to look better in a bikini? An evening dress? Or does she simply want a better, more proportional and shapely appearance in everyday work clothes?

One underemphasized issue is that the surgeon and the patient primarily focus on her breast area with less regard to her overall proportions. Yet there seems to be some regionalized conformity for average breast implant sizes.

Figure 1 displays average implant sizes from various regions and cities.

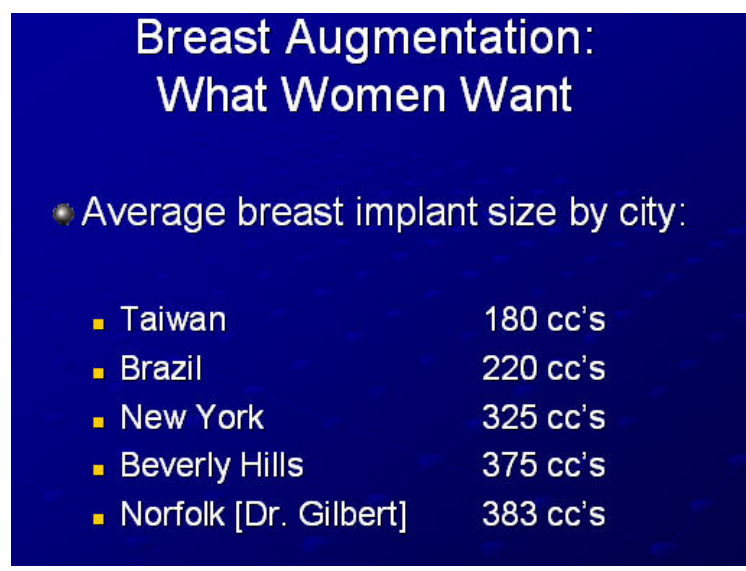

Figure 1. Example Average Breast Implant Sizes by Various Regions/Cities.

My own interviews during preoperative breast augmentation consultations over many years can be summarized in Figure 2.

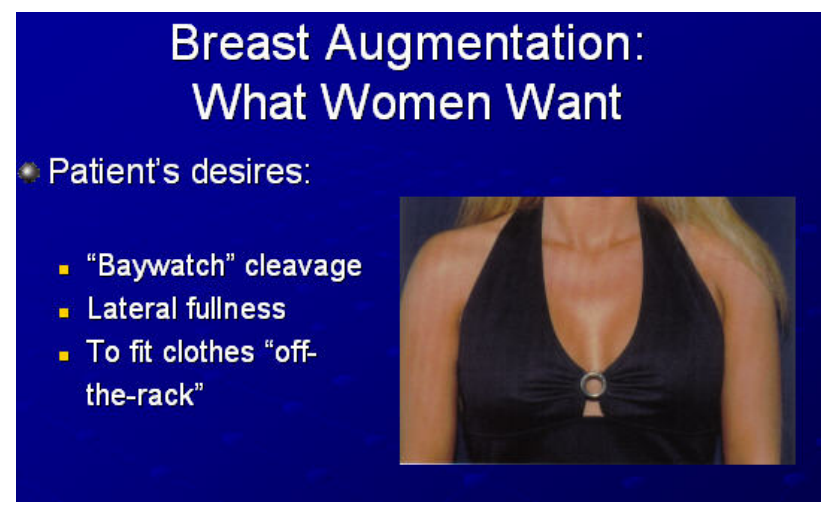

Figure 2. Common Patient Desires after Breast Augmentation Surgery.

As one can see, these are all "value" driven outcomes. A successful surgical outcome, from the surgeon's point of view may not meet the exact objectives of the patient, simply because these objectives are difficult to quantify given that they are perceptions that are not easy to measure.

One common technique for the traditional consultation is to have the patient purchase a bra of her choice in the cup size she believes may be best for her. The patient then supplements the bra by adding false implants or similar devices to fill the bra to the desired size and, depending on the choice of clothing, look.

The patient somehow stops at a point where she feels her breast size may be "too big", then backs down to a desired size.

The surgeon confers with the patient and selects the corresponding implant size and profile to meet the patient's cup size expectations. Gel implants have a higher profile but are more compact. Saline 
implants come in various profiles from low, medium to high. Some saline implants have a valve that can be overfilled to compensate for breast asymmetries.

A general agreement is reached between the patient and the surgeon and the augmentation surgical procedure commences.

In general, the surgeon cannot gauge the "success" of the procedure until swelling abates, which is usually between 3 to 6 months after the surgical procedure. On occasion, it is possible to notice asymmetries earlier given physiological characteristics that may induce uneven settlement.

The patient, however, is likely to evaluate the surgical outcome much earlier. It is during this phase where doubts might arise as to the size of the selection, the particular surgical implementation or even the decision to have undergone the procedure in the first place.

Part of this hesitation, which could lead to an unfounded sense of a disappointing result is due to a lack of an "outcome roadmap" whereby having knowledge of how other implants that she evaluated might have appeared after surgery should she have chosen them.

While many of the surgical outcomes are favorable to both the surgeon and the patient, there are instances where patients indicate they are "happy" with their new breasts, but somehow or other wish they were "bigger", "fuller", or a slightly higher profile.

In extreme cases, this can lead to costly revisions, or worse, a marginal reputation in a community whereby word of mouth is very important.

Based on these experiences, the surgeon needs to have better tools to help engage the patient and develop a bridge between the patient's expectations and the surgeon's capabilities.

Figure 3 outlines, from a surgeon's perspective, some criteria to facilitate this convergence.

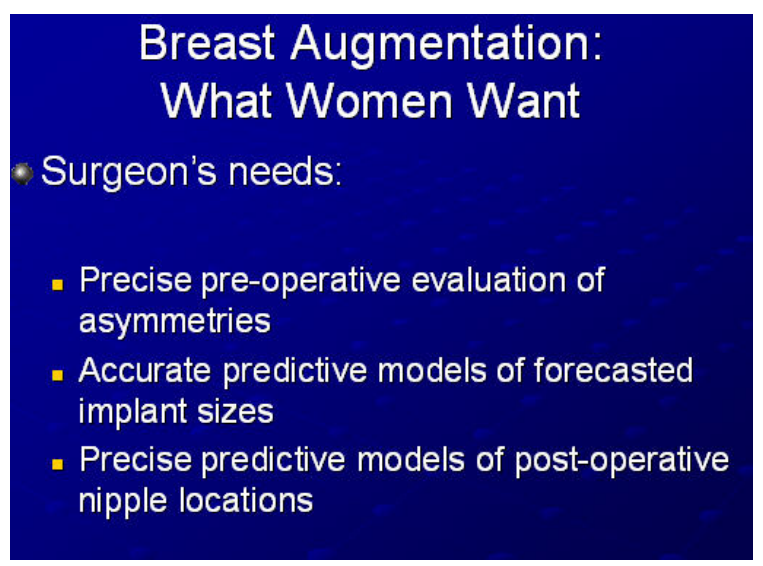

Figure 3. A Surgeon's Wish List

\section{Applying 3D Scanning Technology to Breast Augmentation}

We installed a 3D whole body scanner into the practice in late 2002. At that time, the scanner dimensions were 16 feet by 8 feet. The particular scanner utilized custom-made mechanical projectors and cameras. The scanner consisted of four cameras, and the mechanical projectors would move up and down along a y-axis in front of and in back of the patient capturing various photographs. The technology employed at that time was white light phase profilometry.

These early scanners were impractically large and cumbersome. The mechanical sensors were subject to much maintenance, and the software ran on a version of Windows NT.

Later versions of the scanners collapsed the size, increased the amount of cameras and placed them at fixed length and angles within the scan chamber. The most recent iteration of the scanner has replaced the white light projection/sensor technology with near-field infrared sensors.

The scanner has been utilized in the custom-tailored garment industry. A subject would stand in a fixed position in the scan chamber, the scanning process would commence, and an accurate 3D, measurable body model of the subject would be displayed.

The majority of existing measurement extraction profiles that were available with the scanner were for the purpose of measuring subjects for various garments. One of our first initiatives was to explore the 
scanner software measurement capabilities for suitability in a medical environment. Of particular interest were measurements that focused on the breast area.

Figure 4 displays a scan image of a preoperative breast augmentation patient, an early version of a customized measurement extraction template used for breast measurements, and the resulting measurements.

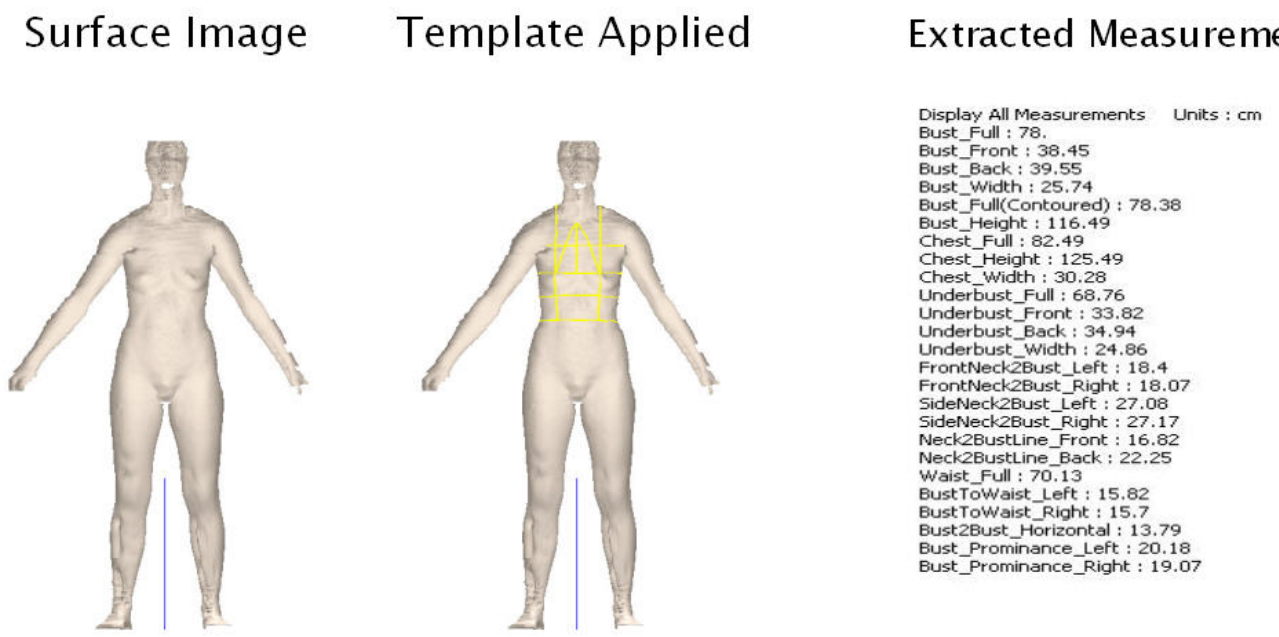

Figure 4. Preoperative Patient and Early Breast Measurement Template.

It was found that many of the measurements selected were automatic measurements with no adjustment capabilities. The few measurements that could be adjusted, such as how the waist measurement was determined had little effect on the breast measurements.

There also was found some variability in the consistency of the scanner measurements, depending on how the patient was standing in the scan chamber. Her breast related measurements would be slightly different if she was standing with her shoulders sloped forward than if she was standing erect in the scan chamber.

Nonetheless, the measurements offered by the scanning software related to the breasts remained intriguing and were explored in further detail.

One important aspect of the scanner was its convenience in simply documenting the patient at a particular point in time.

Figure 5 depicts a series of scans, ranging from preoperative to four months postoperative of a breast augmentation patient.

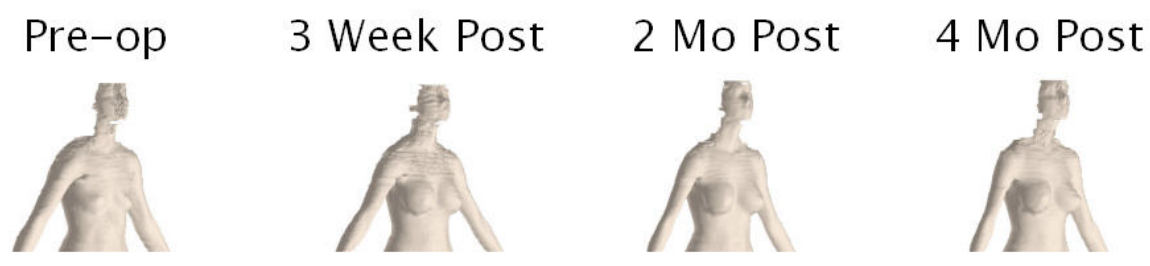

These 3D Images can be rotated freely to view at all angles
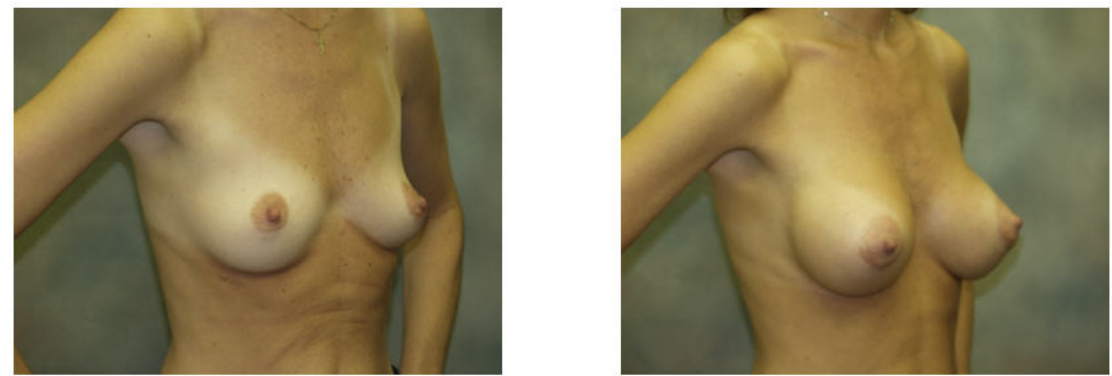

Figure 5. Longitudinal Scan Images and Photos of a Breast Augmentation Patient 
Before 3D scanning, a typical surgeon would document their work by taking analog photographs, then digital photographs as that technology became widely available. This is still done today, but their importance in evaluating surgical results has diminished. This is due to the tremendous advantage that 3D body scanning offers as an archiving tool. Once the patient has been scanned, the 3D body model is saved. Various different measurement profiles can be applied to the saved 3D body model.

Instead of going back, in some cases years, to examine an inconsistent, non-measurable set of photographs, one only has to recall the saved $3 \mathrm{D}$ body model, apply the appropriate measurements and collect and record the information.

Applying the early measurement template sed for breast measurements yielded good results when the appropriate measurement markers were found on the body model. Figure 6 shows such as example.

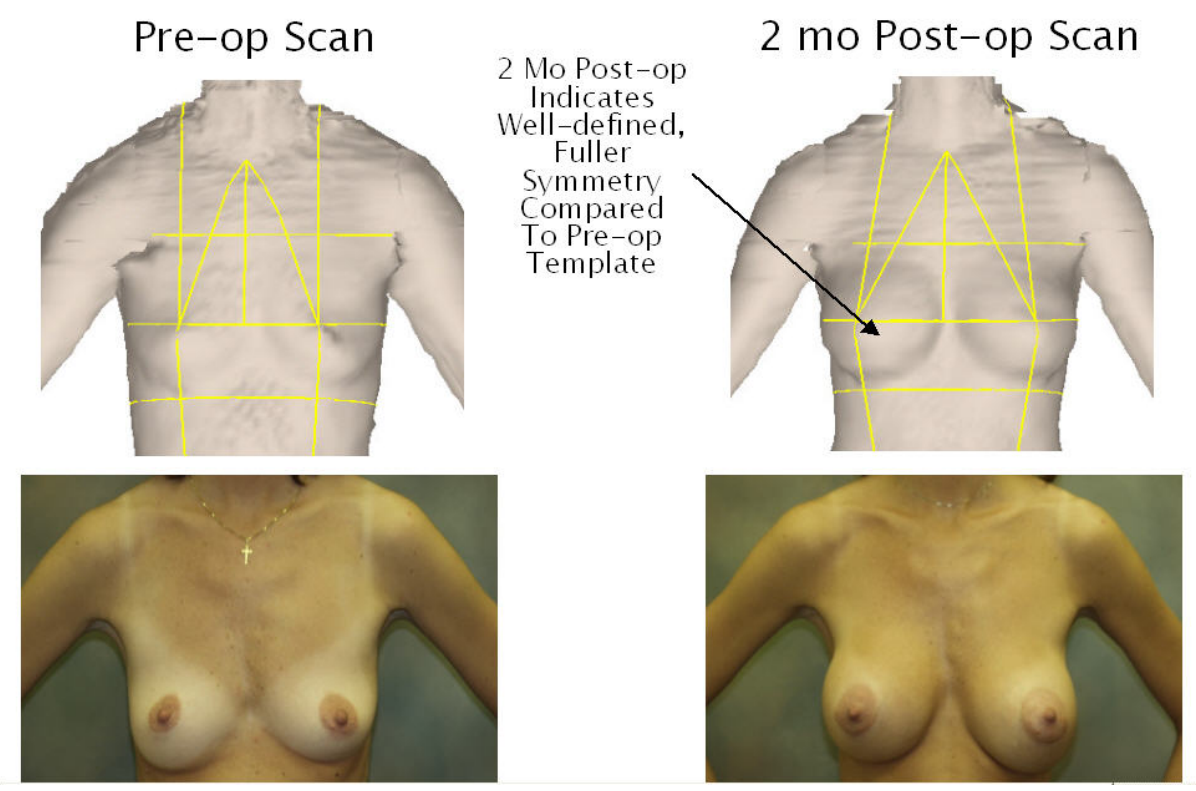

Figure 6. Breast measurement template applied to pre- and postoperative body model

In each case, the automatic measurements appeared to be relatively accurate. The scanner found the subject's nipple locations accurately, and the circumferential bust measurement defined as the maximum circumference of the bust projection appears correct. If one observes the template shape for the post-operative scan, it appears that the resulting implants were symmetrical.

As more cases were documented, it was recognized that the automatic measurement capabilities of the existing scanner software needed to have some manual input to meet the needs of the surgeon. This is highlighted in Figure 7.

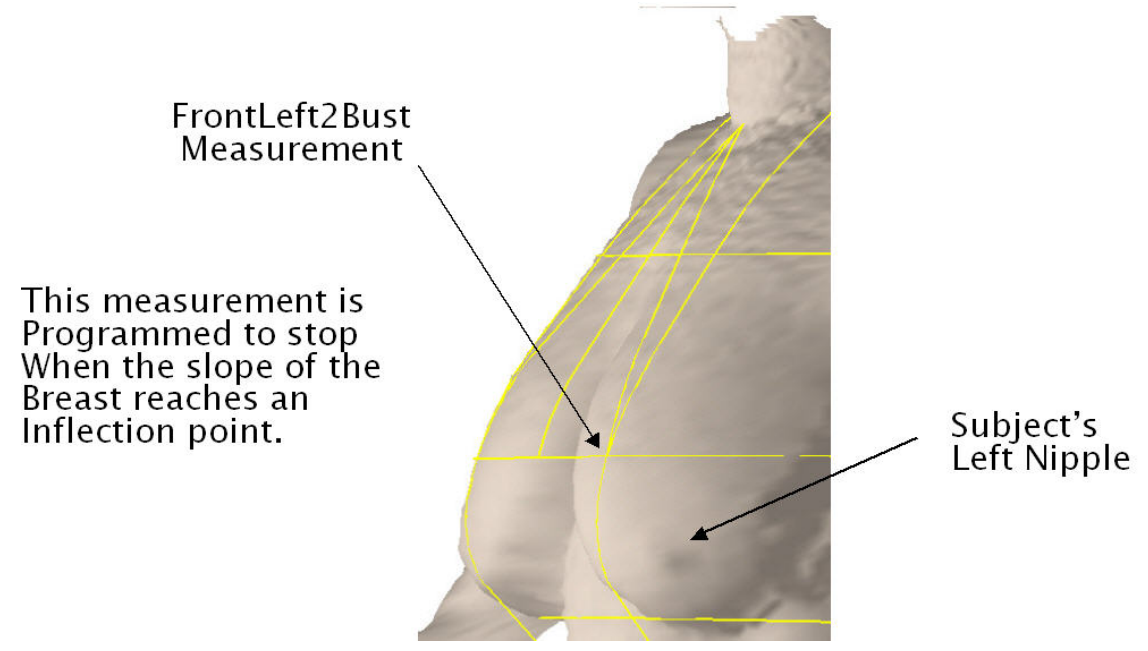

Figure 7. Incorrect Automatic Measurement 
The patient in Figure 7 has large and ptotic breasts. The scanning software's measurement capability for the breast assumes the bust height is at the maximum protrusion of the breasts.

Clearly, the automatic measurement capability that was then current in the scanner software, while helpful overall, was somewhat inadequate for measuring the breasts from a medical point of view.

In 2009 , the scanner manufacturer introduced the capability to manually override certain automatic measurements, including existing measurements associated with the breasts. These included items such as "bust point right and left" which could be used to locate the patient's nipples, and "underbust right and left" measurements that could be manipulated in order to better measure some types of breast asymmetries.

The manual override capability was a great improvement over static automatic measurement extractions, but it soon became clear that the existing bust measurements available as part of the scanner measurement software were inadequate for defining the breast from a medical perspective.

In 2011, a proposal was submitted to the scanner manufacturer for a "moveable points" feature that would overcome the automatic measurements associated with the breasts. This feature added additional measurement points that are specific to the medical community, but of limited or no use for garment sizing.

These new measurement capabilities allowed the surgeon to define the outlines of the breast. Figure 8 displays the nomenclature and measurement locations for these points.

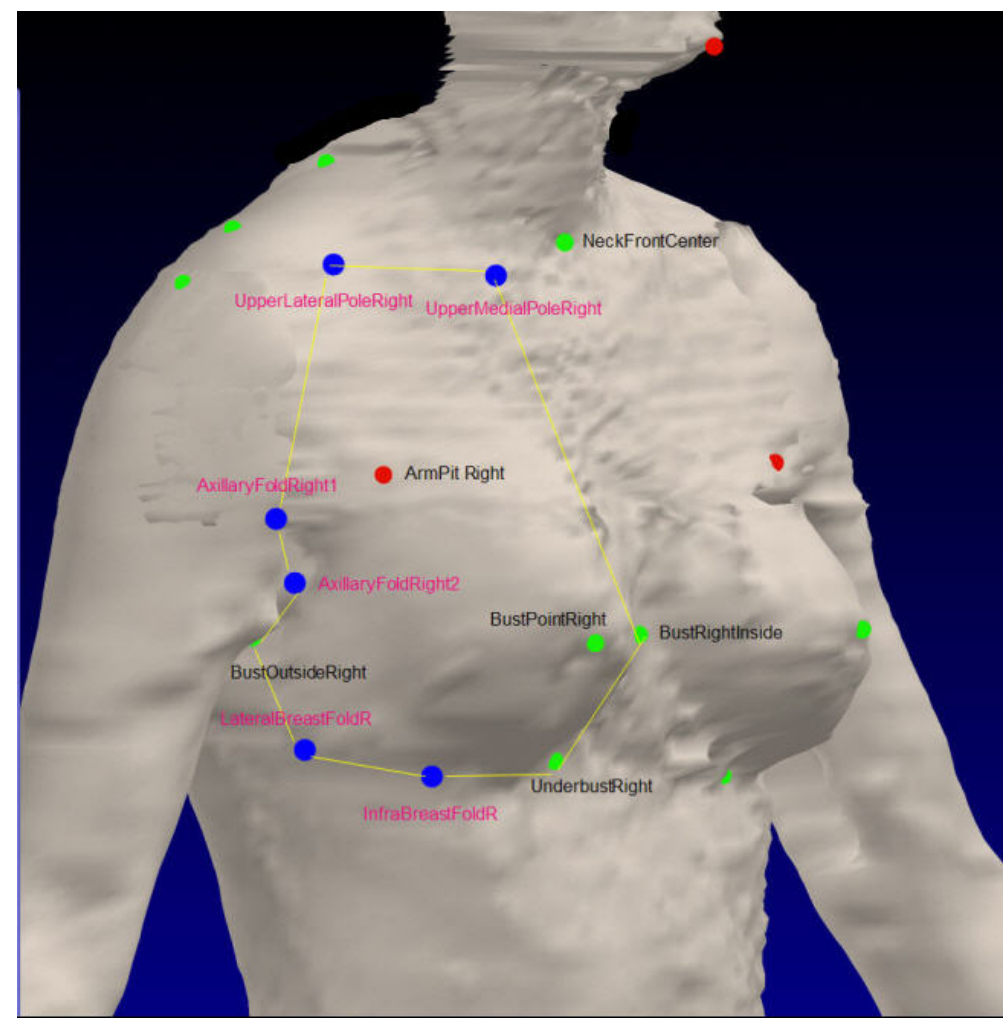

Figure 8. New Manual Measurement Points to Define the Breasts

The labeled points colored green were available breast measurements that could be manually adjusted. The points in blue represent additional manual measurement points required by the surgeon to define the breast dimensions.

Now the surgeon could use his medical training and knowledge of the anatomy of the breast to manually adjust points along the breast folds, which define the perimeter area of each breast. Once adjusted, measurements could be extracted that would accurately determine breast size, including area and volume.

Figures 9 and 10 display the manually adjusted breast measurement template. Figure 9 displays the measurement template on the surface image view of the patient. Figure 10 depicts the same patient in "point cloud" view to better visualize the outline of the breasts and the measurement lines of the breast template. 
Figure 11 depicts the patient and the manually adjusted breast template in supine view, with her head being at the front of the image.

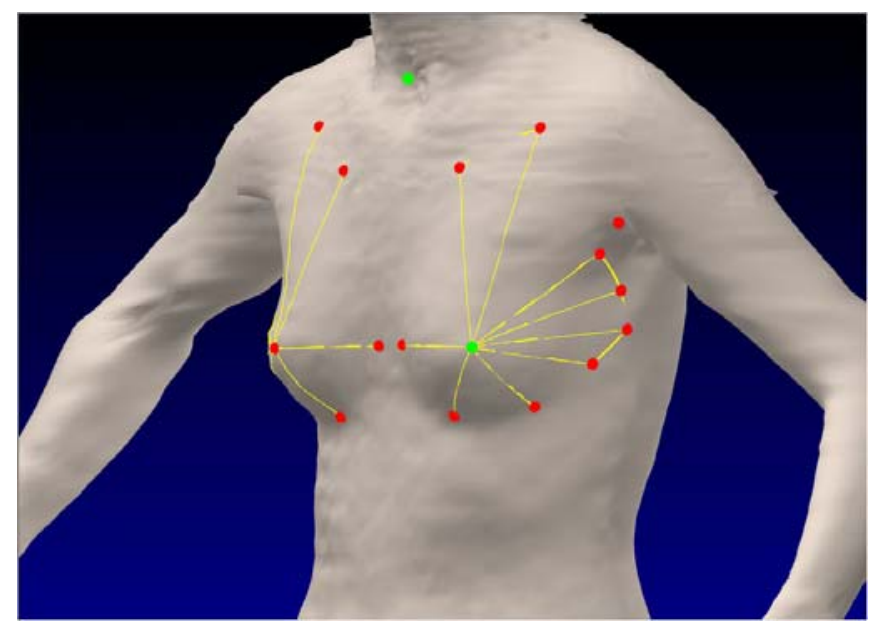

Figure 9. Manually Adjusted Breast Measurement Template - Surface View.

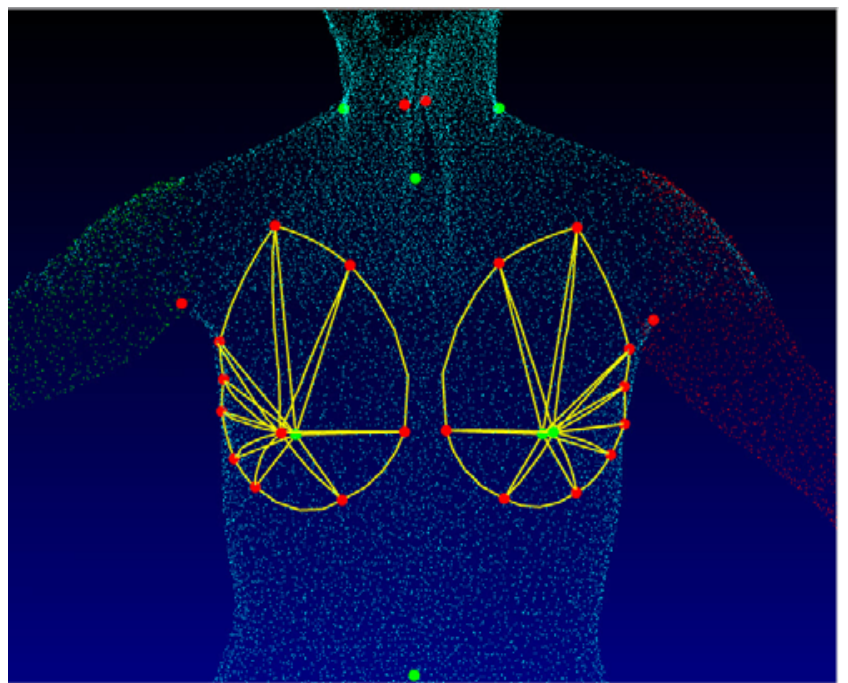

Figure 10. Manually Adjusted Breast Measurement Template - Point Cloud View.

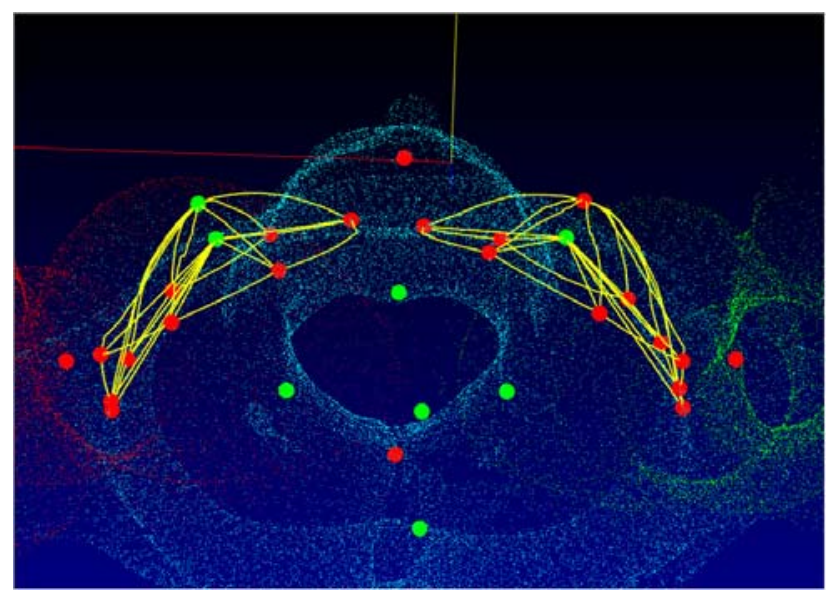

Figure 11. Supine View of Breast Measurement Template - Point Cloud. 
The manual override capability and the new breast point measurements proved to be immensely helpful from a surgical perspective. Appropriately positioned, the measurements extracted from the template helped to identify specific breast asymmetries. Measurement information provided from this template outlined asymmetries along bust contour lines, in $\mathrm{cm}$, along surface area planes, in $\mathrm{cm}$ squared, as well as breast volume in cc.

Identifying and quantifying these asymmetries prior to surgery grants the surgeon the capability to anticipate and overcome them during the surgical procedure.

\section{Predictive Breast Augmentation Modeling}

Now that the scanner software had the capability, via specific measurement point manipulation, to define and measure the breast from a medical standpoint, the 3D body model of the preoperative breast augmentation patient could be accurately documented.

The 3D body model produced by the scanner can be exported to a data modeling software program, in this case via .wrl format. The 3D body model produced by the scanner is ultimately just a collection of points on the surface of the subject. These points are defined in Cartesian coordinates. The 3D body model produced by the scanner consists of seven body parts: The torso, including the head; right and left arms; right and left legs; and right and left hands.

The points along the surface of each body part are connected to form edges and facets which can be deterministically manipulated and measured. If one can accurately model the dimensions, volume and characteristics of a real breast implant to create a set of "virtual implants", one can then "embed" various size implants and expand the breast area of the preoperative body model to create a "predictive model" of what the subject would look like given a particular choice of implant sizes.

Once embedded, this predictive model can be exported out of the modeling program, in .wrl format.

A data converter utility program converts the .wrl predictive model into a raw data format that the scanner can interpret. This raw data file is identical in form to the file produced by the scanning software as if someone was being scanned in the scan chamber. It is then converted into a measurable 3D body model, as if the subject had already undergone surgery.

Thus multiple, accurate $3 \mathrm{D}$ predictive breast augmentation models can be created using various virtual implants sizes that represent the selection of implant sizes that the patient and the surgeon would like to investigate.

The resulting 3D predictive body models can then be measured using various measurement extraction templates. One template would have measurements that the patient can understand, such as bust circumference and bra cup size, along with other whole body measurements such as waist and hips. The other template would be the one that the surgeon can manually adjust to anatomically measure the postoperative breast area in cc.

We have coined the term "Virtual Surgery Cycle" to represent this process. Figure 12 outlines the Virtual Surgery Cycle sequences.

\section{The Virtual Surgery Cycle}

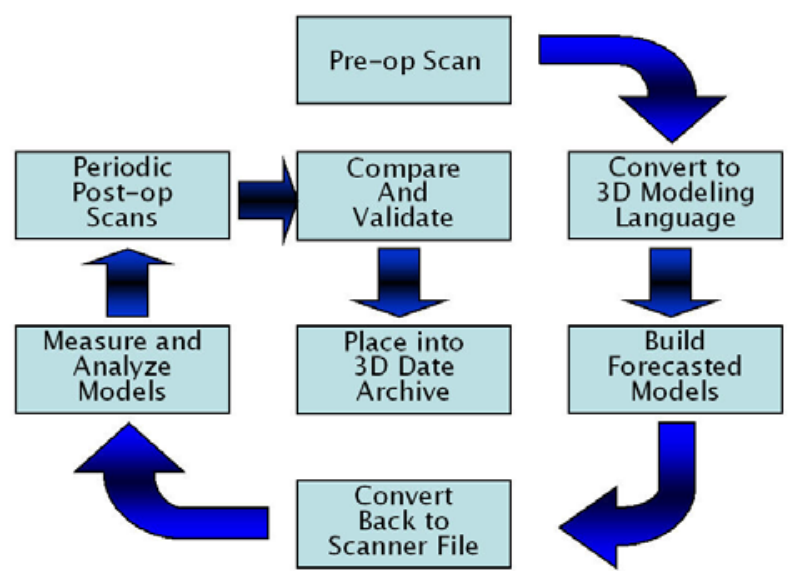

Figure 12. The Virtual Surgery Cycle. 


\section{The Predictive Breast Augmentation Process}

The first step is to export the $3 \mathrm{D}$ body model into a common language that a CAD program can import. In this case, we have chosen the .wrl format. Figure 13 shows such an example.

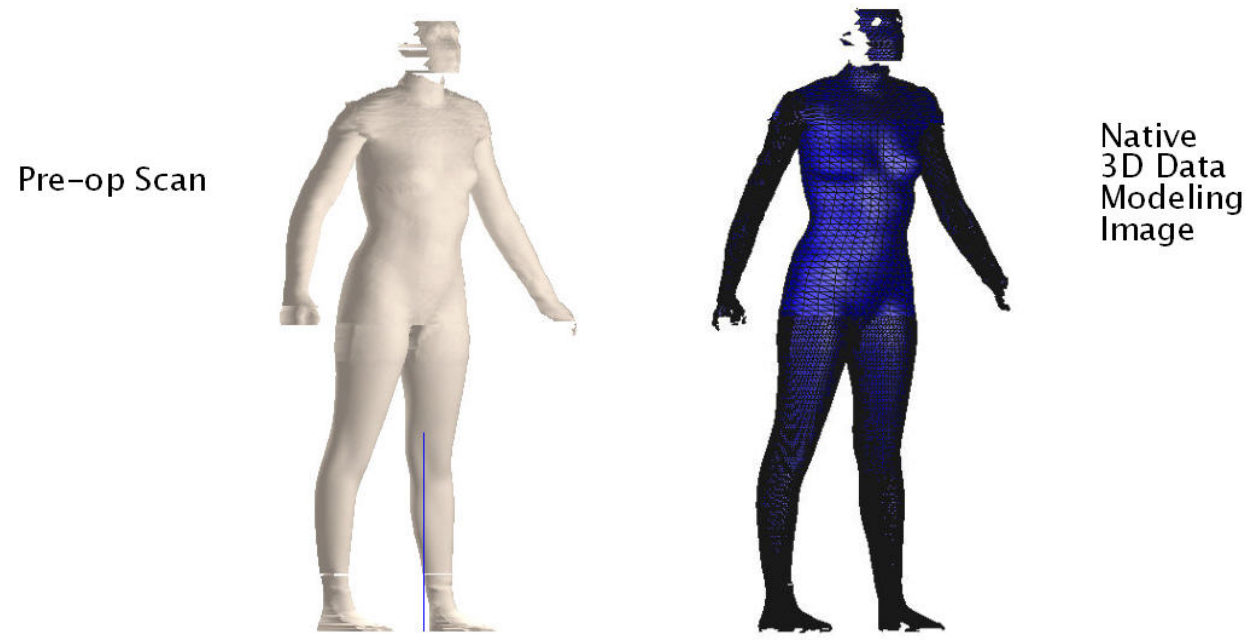

Figure 13. 3D Body Model Exported in .wrl Format.

There are many CAD modeling programs to choose from. The original work was done using AMAPI 3D, which was then purchased by EOVIA, the makers of Carrera. There has been success using several CAD packages, and a great deal of comfort using Cinema 4D and its subsequent follow-on iterations, by MAXON.

The astute reader may recognize the different CAD packages utilized by each illustration's particular characteristics.

Once the model is exported, torso volume is calculated using measurement tools within the particular CAD package. Figure 14 depicts one method of determine torso volume.

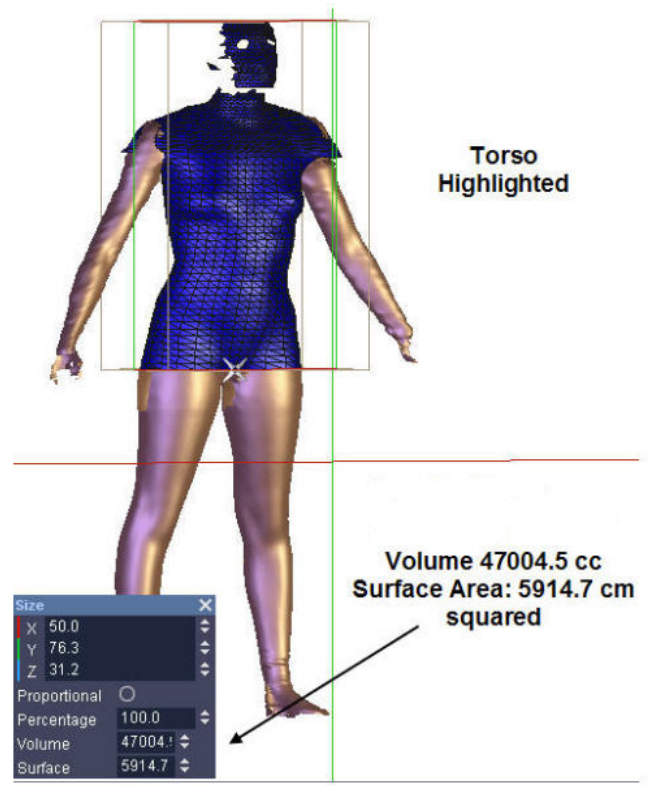

\begin{abstract}
The points, edges and facets of the image form a mathematical representation of the body surface.

Manipulation of these "splines" change the shape of the surface.
\end{abstract}

Changing the shape of the image surface also changes the Volume and Surface Area.

The changes to the image's surface contours can be calculated.

In this way, accurate forecasted models can be developed for various surgical outcomes.

Figure 14. Using CAD Measurement Tools to Determine Torso Volume.

Determining initial torso volume allows the modeler, or "virtual surgeon" to calculate volume changes of the torso as a virtual implant is embedded.

Using a CAD modeling program, one can model a library of "virtual implants" that accurately represent a real implant's dimensions, surface area and volume. This can be done by creating a sphere using the dimensions given by the implant manufacturer, then deforming the sphere into the shape of the implant. 
Typical dimensions are given as width, height and volume. The height determines the profile of the implant. It is important to note that types of implants have certain characteristics that influence the profile. As mentioned earlier, saline implants have a different profile than gel implants.

Figure 15 displays an instance of an accurately modeled virtual implant. In this case, the implant modeled was a Mentor Smooth 1600. Note the accuracy of the dimensions and volume.

\section{Construct Volumetrically and Dimensionally Correct Virtual Breast Implants}

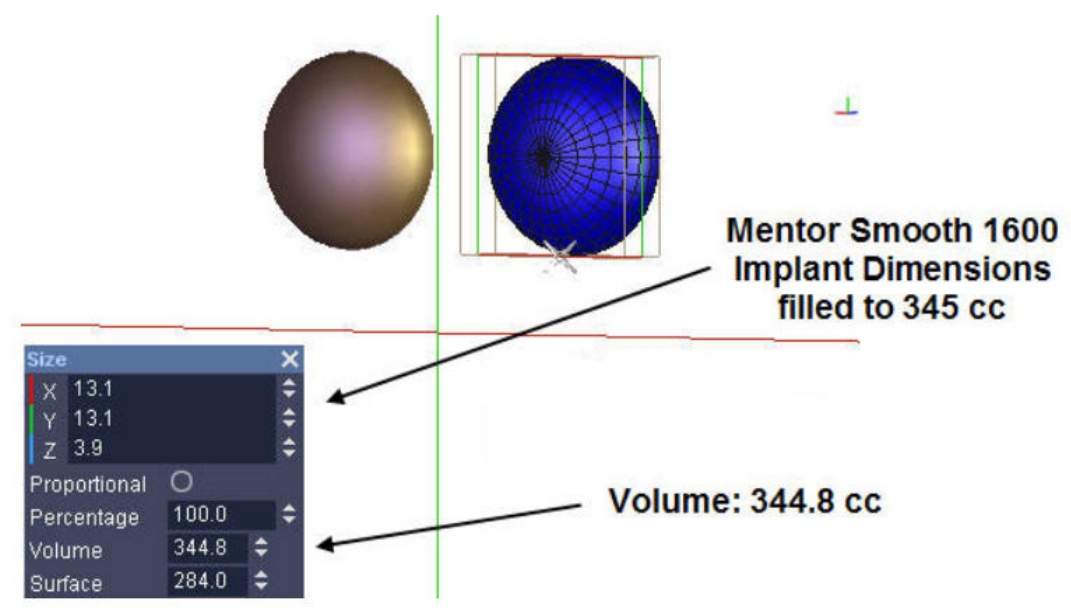

Figure 15. Accurately Modeled 345 cc Mentor Smooth 1600 Implant.

Once the virtual implant has been selected from the virtual implant library, the virtual implants need to be located on the chest wall. The Cartesian coordinates representing the breast augmentation template manually adjusted by the surgeon are helpful in this placement, but for illustration purposes they are not shown.

Figure 16 displays a simplified torso view of the preoperative 3D body model, as well as the location of the virtual implants on the chest wall.

\section{Locating the Virtual Implants on the Images Chest Wall}
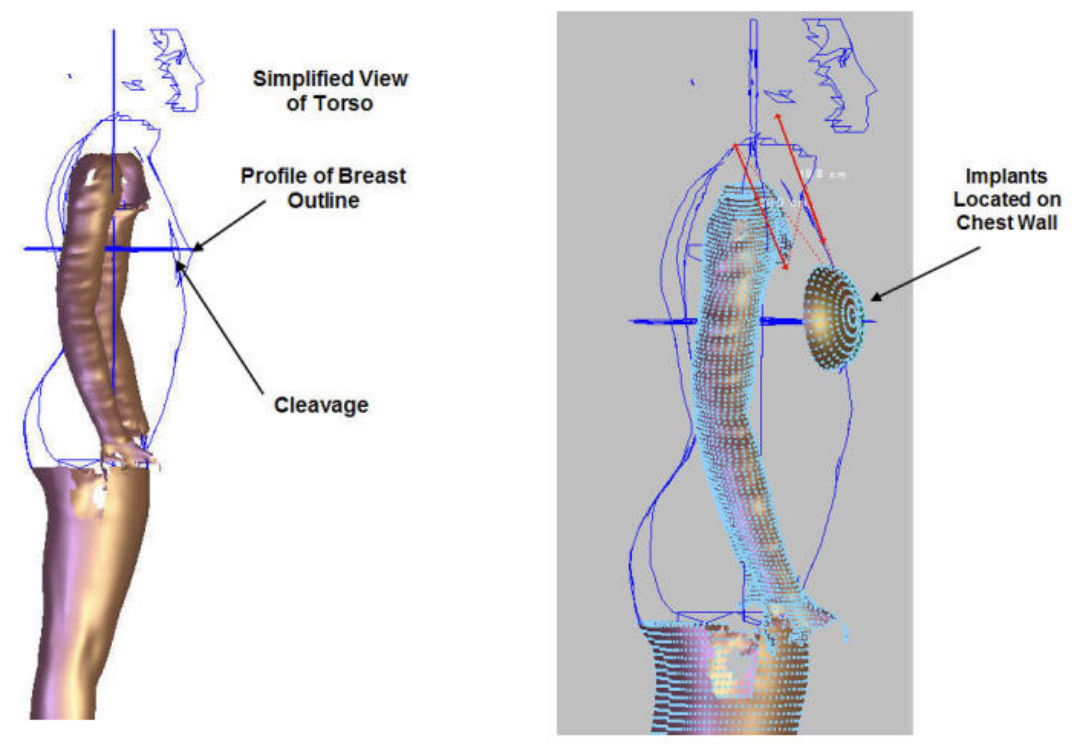

Figure 16. Simplified View of Torso and Location of Implants on Chest Wall. 
Figure 17 depicts a close-up view of implant positioning. Note the existing breast contours of the preoperative patient. One can measure an angle from the center of the 3D body model that represents the patient's nipple projection. The virtual implant must be rotated appropriately to accommodate this angle.

\section{Close-up views of implant positioning}
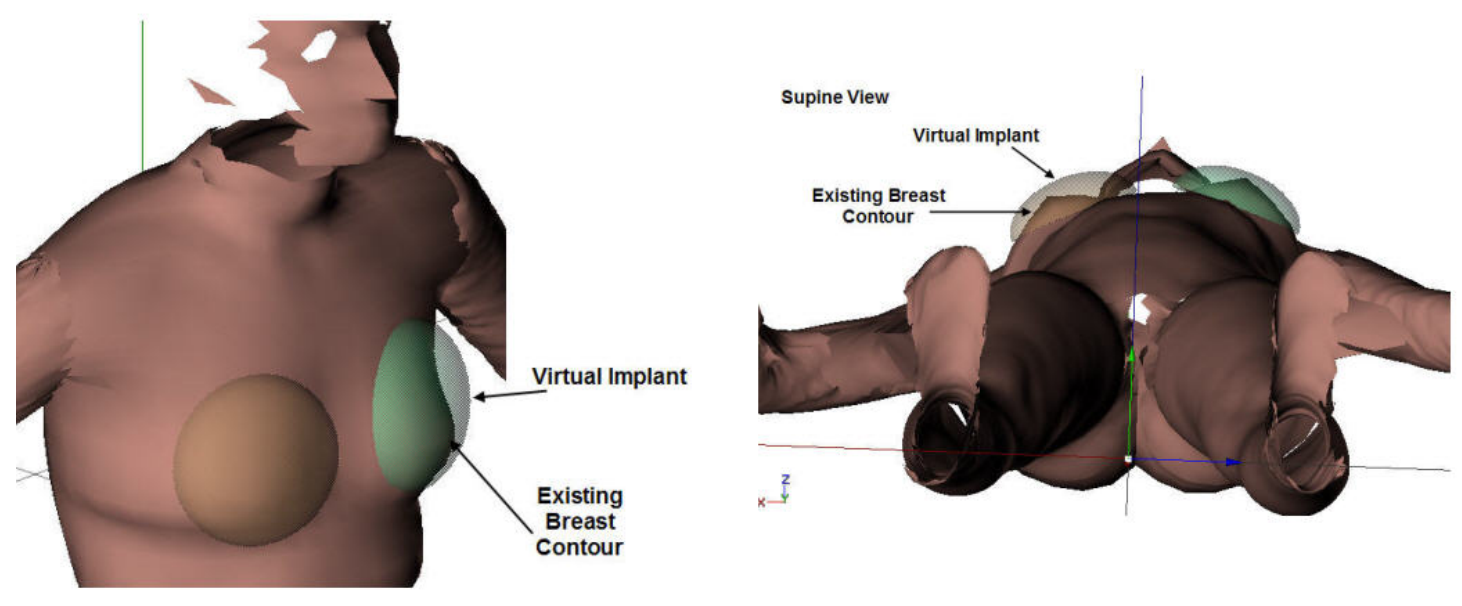

Figure 17. Close-up View of Implant Positioning.

Once the implant is accurately positioned, tools are used to manipulate splines along the breast area. There are methods of using dynamic geometry that can "expand" the chest wall area as the implant is put in place. This is helpful, but often needs to be manually adjusted to account for any preoperative asymmetries.

Figure 18 shows the result of an accurately embedded virtual implant augmenting the left breast.

\section{Embedding the Virtual Implants in the Torso Image}
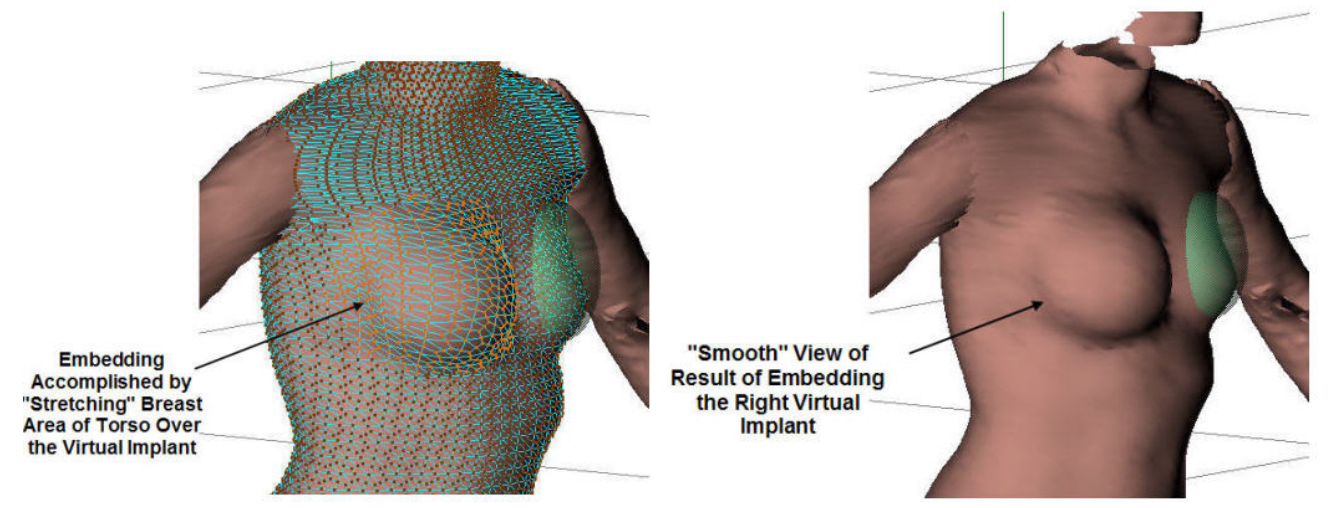

"Stretching" and "Scaling" tools within the Software Suite Manipulate the Torso

Figure 18. Accurately Embedded Left Breast Virtual Implant.

It is important to realize that this embedding process does not graft an implant onto the chest wall, nor does it simply attach it to the surface of the torso. The embedding process needs to take into account that the actual implant is embedded in underneath the breast tissue, and that there is a known separation space representing the nipple complex.

Figure 19 shows the result of the implant embedding process. The torso is displayed in transparent mode so the reader can view the embedded implants and their position within the chest wall. 


\section{Completing the Virtual Breast Implant Embedding Process}
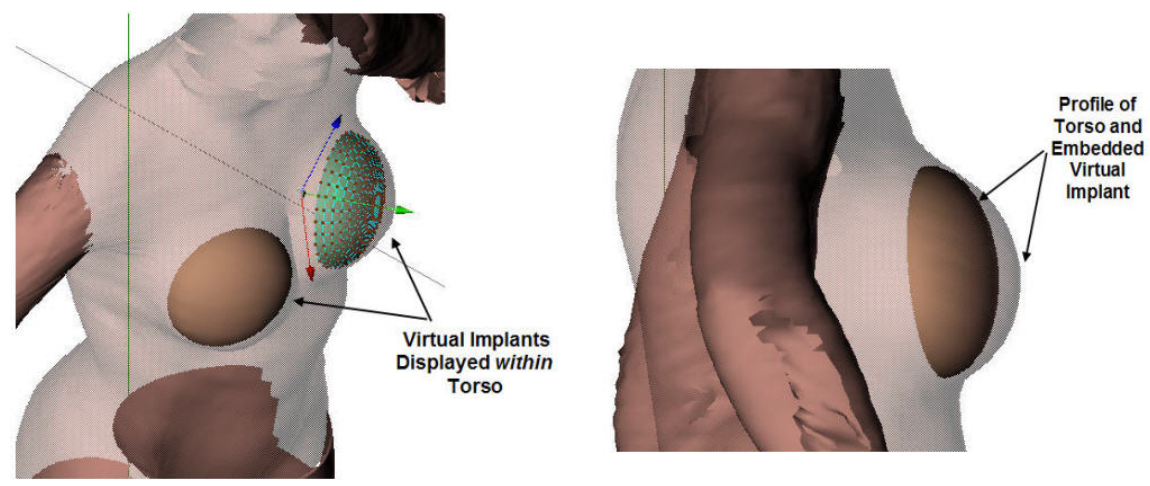

The Space Between Torso and Implant Approximates Tissue/Muscle

Figure 19. The Completed Virtual Breast Implants.

The results of the virtual implant embedding process can be viewed at various angles within the CAD modeling program. Figure 20 shows such as representation.

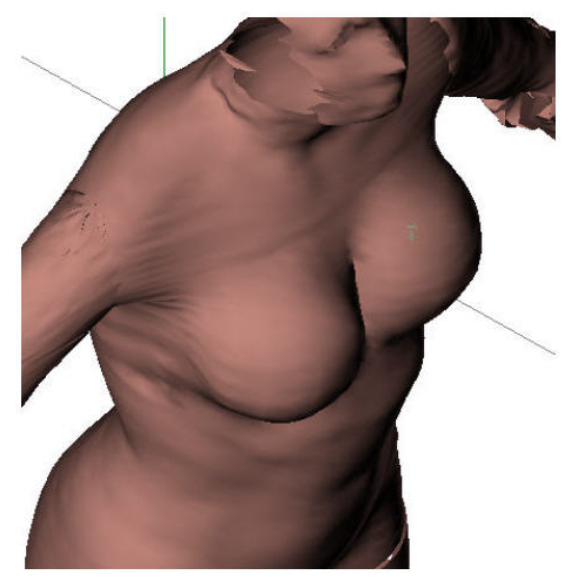

Figure 20. One View of the Embedded Implants.

As part of the embedding process, one can measure the changes in torso area volume that occur after each implant is embedded. Figure 21 outlines the total torso volume changes once both $345 \mathrm{cc}$ implants have been embedded.

\section{Measuring the New Volume and Surface Area}

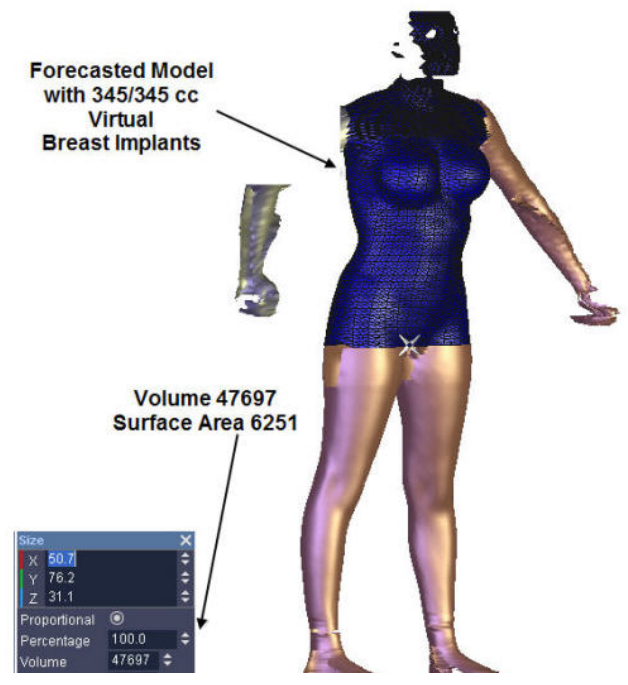
Initial Torso Volume:
$47000 \mathrm{cc}$

345/345 Embedded

Torso Volume: 47697

Volume Change: 697 cc

Figure 21. Measuring Changes in Torso Volume after Embedding Process. 
The total torso volume change was $697 \mathrm{cc}$, very close to the $690 \mathrm{cc}$ one would expect by implanting two 345 cc implants.

Creating accurate predictive breast augmentation models in CAD programs are valuable to the patient only if they can relate to the measurement changes they can expect to have once the surgical procedure has been completed and the associated swelling has abated.

In order to create a measurable $3 \mathrm{D}$ body model from the predictive CAD model, the predictive CAD model is exported in .wrl format.

A data converter utility is employed to convert the predictive CAD model from .wrl format to a .bin format, which is the raw data format the scanner software uses to create a measurable $3 \mathrm{D}$ body model from raw data camera images.

Figure 22 represents the predictive 3D CAD model, and its associated conversion to a measurable 3D body model within the scanner software.

\section{Translating the Forecasted Model Back to Scanner Format}

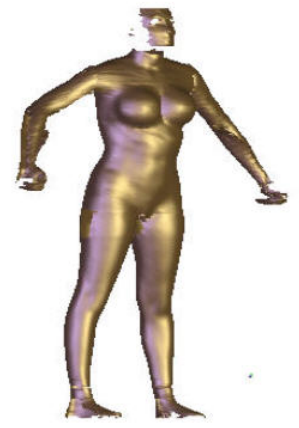

Forecasted Model

In 3D Modeling Language

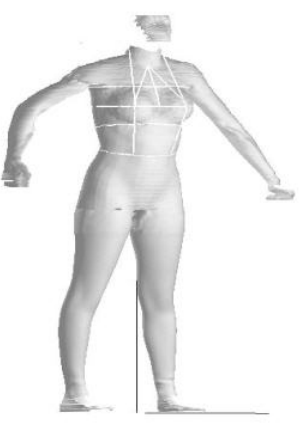

Forecasted Model

Converted to

Scanner Format

Figure 22. Forecasted Model in CAD Format Translated to Scanner Format.

The forecasted, predictive model is only as good a guide as its accuracy against the actual measurements of the postoperative patient, but the validation is straightforward. The same measurement template can be used to measure the actual postoperative body model of the patient and the forecasted model, assuming that the effect of swelling from the surgical procedure has abated.

Figure 23 shows such validation.

\section{Comparing Measurements between Forecasted Model and Post-op Scan}

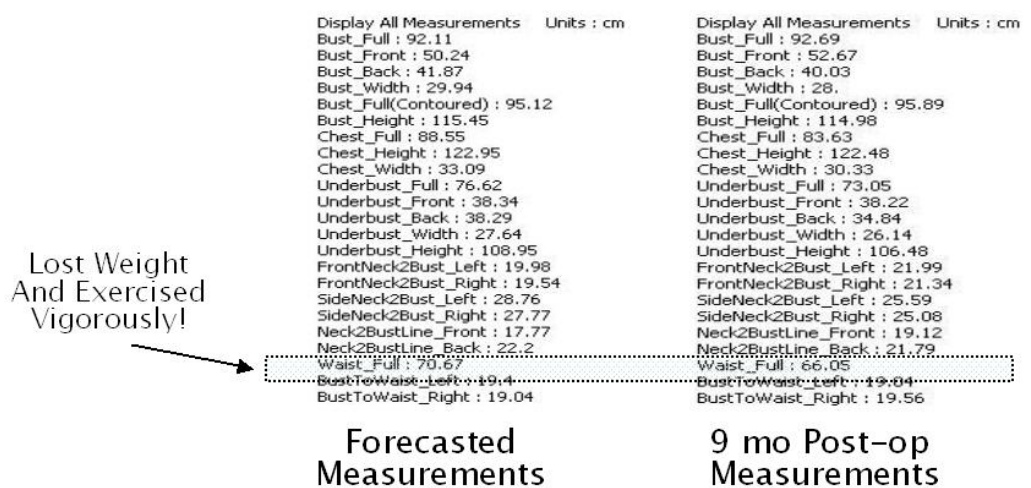

Figure 23. Validation of Forecasted Model to 9 Mo Postop Patient Scan, units in $\mathrm{cm}$.

Of course, for the American audience, the measurements would be in inches. Moreover, the standard clothing templates associated with the scanner's garment measurement capability can be invoked, including bra cup size. 
The breast augmentation template manually created by the surgeon can also be used to validate the predictive model with the actual post-operative model.

Through the use of the scanner, and through the use of common measurement templates and customized, manually placed breast measurement points, a method of creating accurate predictive breast augmentation models now exists. Validation can be done on these predictive breast augmentation models by converting them to the scanner format and measuring them using templates that the patient and the surgeon can individually understand.

\section{The New Breast Augmentation Consult - Patient and Surgeon Engagement}

Implementation of 3D scanning technology and its ability to create an accurate, preoperative body model, and measurable predictive augmentation models now allows the surgeon and the patient to have a different level of conversation during the consultative process.

Figure 24 shows what we have termed "The New Breast Augmentation Consultation Process".
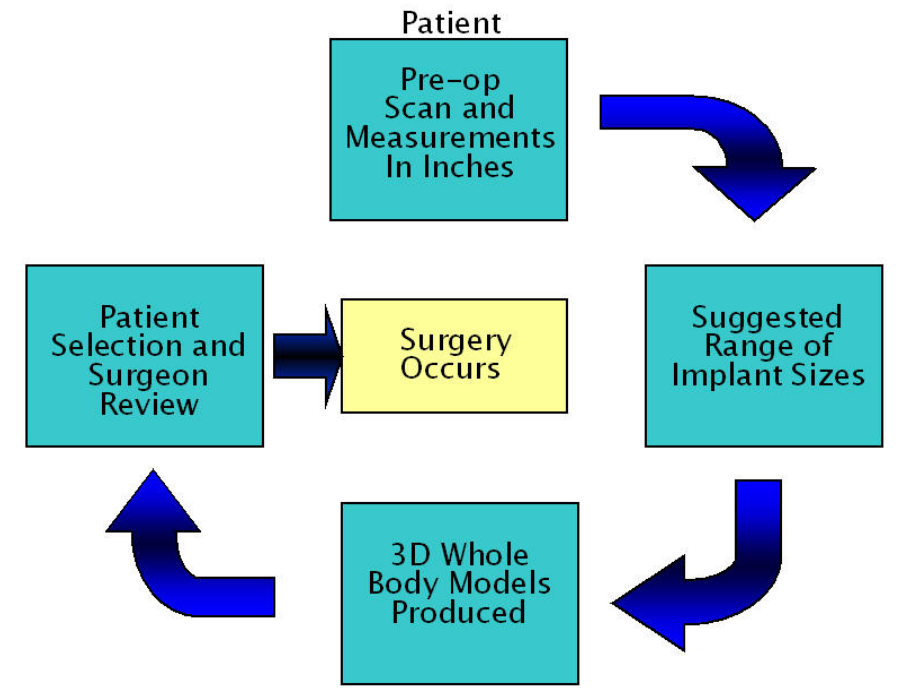
Surgeon

Figure 24. The New Breast Augmentation Consultation Process.

Because the 3D scanner produces whole body models, a patient can select multiple implant sizes, have them modeled and measured and view what these potential implant sizes could look like in overall proportion to her body.

Figure 25 shows 3D composite images of a subject who chose $400 \mathrm{cc}, 436 \mathrm{cc}$ and $475 \mathrm{cc}$ implants sizes as potential implants for a pending breast augmentation procedure.

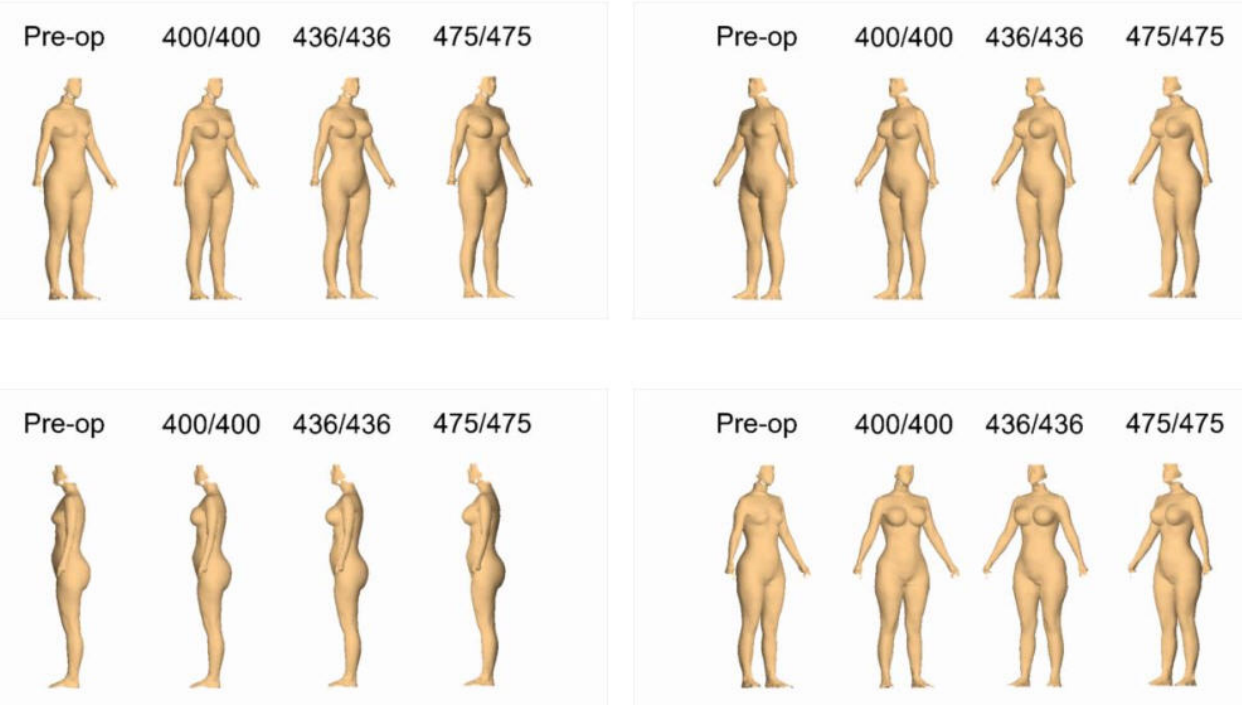

Figure 25. Composite Predictive 3D Images of Various Implant Sizes. 
Each model has its own specific measurements. The patient can now visualize her potential appearance and choose the breast implant size that best reflects the image of how she would like to appear after the surgical process.

The fact that the patient can now actively engage in implant selection, given that she has a potential understanding of the surgical outcome, has set a new level of patient expectations. There appears to be better acceptance of the surgical outcome, all things considered. This is perhaps because the patient chose the particular implant size, with some knowledge of the potential outcome, and has therefore taken more responsibility than she would have had otherwise had she not had this "outcome roadmap".

\section{What's on the Horizon?}

The next logical step is to explore answering questions such as "What would I look like in an evening dress with these implant sizes"? Or, "How would I look like in a "clubbing outfit" from the viewpoint of a someone sitting at a table"?

These may seem like outlandish questions at first. And they were a number of years ago before the advent of 3D scanning technology.

Now it is possible to select clothes from a "Virtual Closet" and shop for garments online.

The following are two hypothetical illustrations using a preoperative patient who wished to be modeled using $500 \mathrm{cc}$ saline implants. Figure 26 shows her predictive model in an evening dress she selected from a clothing manufacturer.

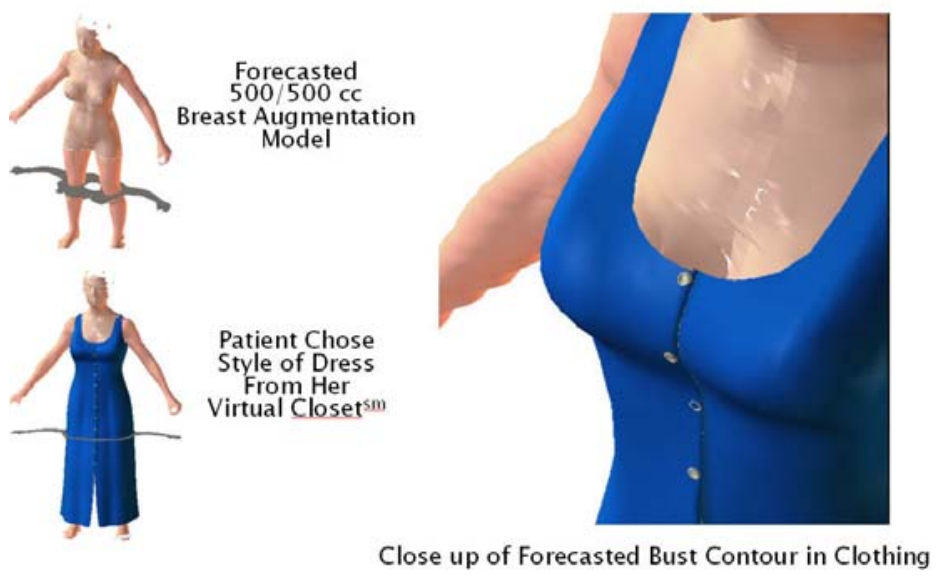

Figure 26. A Predictive Breast Augmentation Model with Clothing.

Figure 27 answers her question about how she would look walking by a table in a "clubbing outfit".
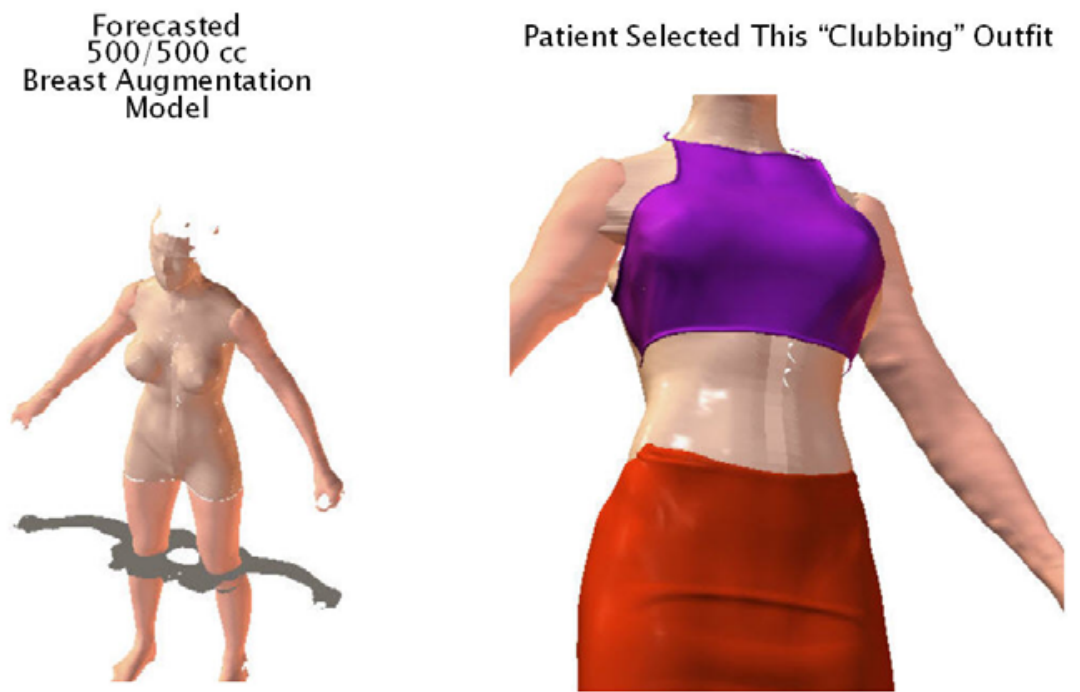

Figure 27. Multiple Clothing Options for Predictive Model. 


\section{Conclusion}

3D body scanning has opened up new avenues of communication between the patient and the surgeon. It has done so by bridging a language gap. The bridge is the fact that the patient and the surgeon both want a successful outcome, but until the accurate predictive modeling capability, they viewed a successful outcome in different ways.

The scanner and its manual measurement capability address the key points required by the surgeon in order to be an effective pre- and post operative breast augmentation evaluation tool.

By creating predictive models, and extracting measurements that the patient can understand, such as bust circumference and bra cup size, the issues of doubt about implant size selection can start to be overcome.

Thus by helping the surgeon and the patient to visualize potential outcomes from two different perspectives, the end goal of patient satisfaction and successful surgical outcome converge. 